\title{
Rheological properties in solutions of wormlike micelle composed of lysophosphatidylcholine/phosphatidylcholine mixture
}

\author{
Paphawee Nantarajit $^{1}$, Natdanai Fafaungwithayakul ${ }^{2}$, Natsuda Vichakacharu ${ }^{1}$, \\ Shingo Matsukawa ${ }^{1, *}$ \\ ${ }^{I}$ Department of Food Science and Technology, Tokyo University of Marine Science and Technology, 4-5-7 Konan, Minato, \\ Tokyo 108-8477, Japan \\ ${ }_{2}^{2}$ SMS Corporation, 38/6 Moo 11, Lat Lum Kaeo, Pathum Thani 12140, Thailand
}

Received: 29 July 2017 / Accepted: 7 November 2017

(C) Japanese Society of Biorheology 2017

\begin{abstract}
We have elucidated the heat induced formation of wormlike micelles in the mixture solutions containing two different geometric amphiphiles of lysophosphatidylcholine (LPC) and phosphatidylcholine (PC), where LPC and PC have one and two alkyl chains as hydrophobic groups, respectively. The mixture solutions showed the increases in viscosity and transparency by heating, and showed the highest viscosity at the ratio of $\mathrm{LPC} / \mathrm{PC}=1.67$, suggesting the formation of long wormlike micelles attained by the average $c p p$ around 0.5 favorable. From the dynamic rheological measurements, it was found that the elasticity was proportional to the square of total concentration of LPC and $\mathrm{PC}$, indicating the existence of entanglement among long wormlike micelles. Other mixture solutions seem to be mixtures of spherical, lamellar and wormlike micelles with different sizes judging from the viscoelastic behavior and transparency, where the sizes changed reversibly with the temperature changes.
\end{abstract}

Keywords phosphatidylcholine, lysophosphatidylcholine, wormlike micelle, viscoelasticity, gel-like fluids

\section{Introduction}

Surfactants are capable in forming aggregation of various self-associated structures when their concentrations are above the critical micelle concentration (CMC). Self-assembled structures are varied in a range of dimensional defined structures, e.g. bilayers, spherical micelles, cylindrical micelles, or vesicles, where the geometric parameters play an important role to determine the structure as stable state. The

*E-mail: matsukaw@kaiyodai.ac.jp critical packing parameter ( $c p p$ ), is widely used to express the geometry of surfactants, which is written as follows.

$$
c p p=V /\left(a_{0} l_{c}\right)
$$

where $\mathrm{V}$ is the volume of hydrophobic group region, $a_{0}$ is optimal surface area per headgroup at the interface and $l_{\mathrm{c}}$ is the length of hydrophobic region [1]. The transition of amphiphilic aggregates to various morphologically selfassemblies can be triggered by many considerable factors, e.g., molecular size and geometry, intermolecular interaction, composition of surfactants in the system, temperature, application of shear forces and so on [1-4].

Phosphatidylcholine (PC) is the major component found in egg yolk lecithin which is in a group of phospholipids containing phosphate polar head group as hydrophilic region and two strains of fatty acid as hydrophobic region. LPC is a derivative of PC by specified enzymatic hydrolysis to obtain a remained one fatty acid chain, which enhances the hydrophilicity of the molecule [5]. PC and LPC are zwitterionic amphiphiles which have different $c p p$ values and form different self-assemblies in water [1]. PC has double hydrocarbon chains and forms planar structure and lamellar phase, on the other hand, LPC has a single hydrocarbon chain and prefers to form the spherical micelle [1, 5-7].

Mixture aqueous solutions of phospholipids with suitable hydrophilic amphiphiles can form mixed micelles under the appropriate conditions [8,9]. When lipids bilayers were mixed with lipids forming spherical micelle, the various types of micellar structures were formed such as vesicles and micelles depending on the proportions in the mixture solution [10]. The mixture of phospholipids with surfactants undergoes the structural transition depending on surfactant- 
to-phospholipids ratio for different type of surfactants [11]. The micellar structure changes in mixture solution of phospholipids were associated with the interaction among their molecules. The addition of LPC to PC bilayers decreased the rigidity of the bilayers, and up to the certain amount of LPC content which caused the interaction of LPC and PC that can breakup of planar structure of PC and micellar structure can be formed [12]. The aqueous solution of mixture LPC and PC was able to form an elongated self-assembly by heat induced process, and the wormlike micelle was considered to be formed by co-micellization of LPC and PC in the mixture solution [13].

Wormlike micelles are very expedient and have been applied in variety of surfactants applications such as household and personal care products [14]. Additionally, the enhancement of viscosity by sol to gel transition upon heating has been attracted and seem to be applied as potential material in biomedical applications [15]. Since extraordinary viscoelastic behavior of wormlike micelle formation plays an important role for design and formulation of various useful surfactant applications, understanding of the related structural and rheological characteristic of wormlike micelle is important in its application uses as well as fundamental studies. In this work, we elucidated the effect of mixing ratios of LPC and PC on the wormlike micelle formation and rheological properties by dynamic rheological measurements.

\section{Materials and Methods}

\section{Materials}

Egg yolk phosphatidylcholine (PC, $\geq 98 \%$ ) and egg yolk lysophosphatidylcholine (LPC) were kindly supplied by Kewpie Corporation (Tokyo, Japan). PC and LPC aqueous solutions were separately dispersed in deionized water at 64 $\mathrm{mM}$ using high shear mixer (Silverson L5M-A, Buckinghamshire, England) by $10,000 \mathrm{rpm}$ for $10 \mathrm{~min}$ at $40^{\circ} \mathrm{C}$, and mixed with various mixing ratios of $\mathrm{LPC}$ and $\mathrm{PC}$ at 10,000 rpm for $1 \mathrm{~min}$ at $40^{\circ} \mathrm{C}$ by high shear mixer. The aqueous mixture solutions with different LPC/PC molar concentration mixing ratios of $32 / 32,36 / 28,40 / 24$ and $44 / 20 \mathrm{mM}$ in the total fixed concentration at $64 \mathrm{mM}$ were named as $\mathrm{M} 32 / 32$, M36/28, M40/24 and M44/20, respectively. The mixture solutions were heated at $80^{\circ} \mathrm{C}$ for $30 \mathrm{~min}$ in order to induce the micellar transition to wormlike micelle [13]. Afterward, the mixture solutions were cooled down and kept at $20^{\circ} \mathrm{C}$ for $24 \mathrm{~h}$ to ensure the equilibration of micelle formation prior to the measurements.

\section{Rheological measurements}

The mixture solutions of various mixing ratios of LPC and $\mathrm{PC}$ were kept at $20^{\circ} \mathrm{C}$ for $24 \mathrm{~h}$ to equilibrate the micellar structure formation, and then placed at various temperatures of $5,10,20$ and $30^{\circ} \mathrm{C}$ for $24 \mathrm{~h}$.

The rheological measurements of LPC/PC mixture solutions were performed using stress-controlled rheometer (Thermo scientific HAKKE MARS II, Massachusetts, USA) equipped with cup and bob geometry, with a cup (inner diameter $=27.2 \mathrm{~mm}$ ) and bob $($ diameter $=25.1 \mathrm{~mm})$. The mixture solutions were equilibrated at specified temperatures for at least 10 minutes prior to the measurements. The steady rheological measurement was employed with a range of shear rate $(\dot{\gamma})$ from 0.01 to $100 \mathrm{~s}^{-1}$. The dynamic rheological measurements were performed with dynamic oscillatory measurements in the linear viscoelastic regime. The stress sweep measurements were carried out to determine the linear viscoelastic regime, and subsequent frequency sweep measurements under a range of angular frequency $(\omega)$ from 0.01 to $100 \mathrm{rad} \cdot \mathrm{s}^{-1}$ were performed with a chosen critical stress $\left(\sigma_{c}\right)$ which could maintain the network structure.

\section{Results and Discussions}

\section{Temperature dependence of turbidity and viscosity in mixed} $L P C / P C$ solutions

$64 \mathrm{mM}$ PC solution was turbid due to lamellar phase made of planar structures, on the other hand, $64 \mathrm{mM} \mathrm{LPC}$ solution was transparent because it formed spherical micelle. The $64 \mathrm{mM}$ aqueous mixture solutions with various ratios of LPC and PC (M32/32, M36/28, M40/24 and M44/20) showed the turbidity with the degree corresponding to the content ratio of $\mathrm{PC}$ in the mixture solutions as shown in Fig. 1(a). After heating at $80^{\circ} \mathrm{C}$ for $30 \mathrm{~min}$, the turbidity of mixture solutions were markedly decreased due to the changes of micellar structures upon heating. After subsequent cooling to $20^{\circ} \mathrm{C}$ and storing at different temperatures, the mixture solutions changed in the turbidity and viscosity depending on the mixing ratio of LPC/PC and the storage temperature as shown in Fig. 1(b)-(e). Moreover, the LPC/PC mixture solutions in various mixing ratios changed in their viscosities and turbidity with temperature dependence. M40/24 and M44/20 became transparent solutions at all storage temperatures indicating a disruption of lamellar structures of PC and the formation of wormlike micelles which decreased the light scattering of mixture solutions. M40/24 exhibited gel-like behavior at 30 and $20^{\circ} \mathrm{C}$ as it could hold its own weight in an upside-down vial, and the viscosities drastically decreased with decreasing temperature lower than $20^{\circ} \mathrm{C}$ showing changes from gellike fluid to slimy gel which dropped down in the upsidedown vial. Similarly, M44/20 formed a transparent gel-like fluid at $30^{\circ} \mathrm{C}$ and changed to the slimy gel with decreasing temperature from 20 to $5^{\circ} \mathrm{C}$. On the other hand, $\mathrm{M} 36 / 28$ and M32/32 changed to slightly turbid solutions at 30 and $20^{\circ} \mathrm{C}$, and became highly turbid viscous solutions with decreasing temperature. The viscosities of M36/28 and 
M32/32 increased with decreasing temperature, especially the viscosity of $\mathrm{M} 36 / 28$ largely increased at $20^{\circ} \mathrm{C}$ and became highly turbid and viscous suspension at $5^{\circ} \mathrm{C}$.

M40/24 and M44/20 changed to transparent solutions and exhibited gel-like behavior, indicating the presence of wormlike micelles and micellar network by entanglement of wormlike micelles, as seen in our previous work [13]. Although M40/24 and M44/20 were transparent through all range of temperature, they changed in viscoelastic behavior with decreasing temperature indicating a change in the micellar network structure made of the entanglement of wormlike micelles at low temperature. M40/24 and M44/20 remained as transparent slimy gels at low temperature suggesting that the entanglement of wormlike micelles were decreased because of breaking of the long wormlike micelles to short wormlike micelles and/or the formation of spherical micelles which diluted the concentration of wormlike micelle. M36/28 was heterogeneous in turbidity at $30^{\circ} \mathrm{C}$, where higher turbid phase contained more lamellar structures, and became more homogenously transparent

(a)

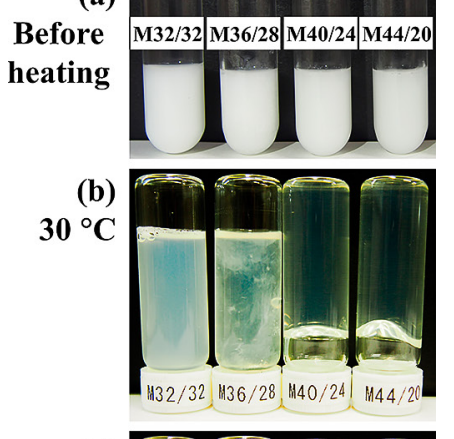

(c)
${ }^{\circ} \mathrm{C}$

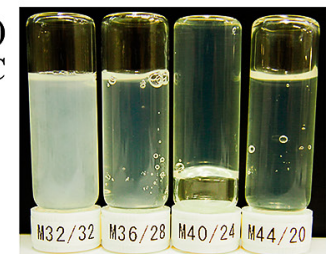

$10^{\circ} \mathrm{C}$
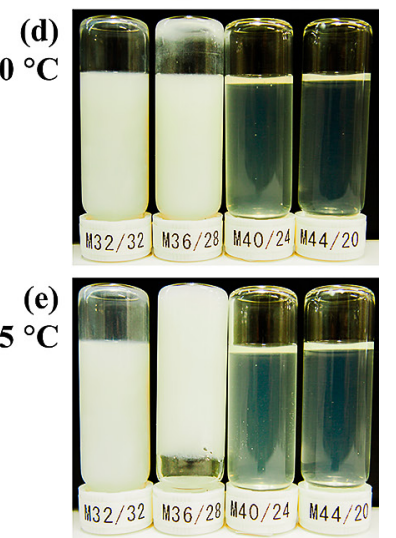

Fig. 1 Photographs of $64 \mathrm{mM} \mathrm{LPC/PC} \mathrm{mixture} \mathrm{solutions} \mathrm{with} \mathrm{different}$ mixing ratios of $32 / 32,36 / 28,40 / 24$ and $44 / 20$, before heating (a) and stored at $30^{\circ} \mathrm{C}(\mathrm{b}), 20^{\circ} \mathrm{C}(\mathrm{c}), 10^{\circ} \mathrm{C}(\mathrm{d})$ and $5^{\circ} \mathrm{C}(\mathrm{e})$, for $24 \mathrm{~h}$ after heating at $80^{\circ} \mathrm{C}$ for $30 \mathrm{~min}$ and subsequently stored at $20^{\circ} \mathrm{C}$ for $24 \mathrm{~h}$. slimy gel at $20^{\circ} \mathrm{C}$ due to the breakdown of lamellar structures and the formation of wormlike micelles. With further decreasing temperature, however, M36/28 changed to a highly turbid and viscous suspension at $10^{\circ} \mathrm{C}$, and to a higher viscous suspension at $5^{\circ} \mathrm{C}$ as it could hold its own weight in the upside down vial and flowed down slowly. From these results, it was considered that the turbidity of M36/28 at low temperature came from the regeneration and expansion of lamellar structures, and the increase in viscosity was caused by the formation of micellar network from the interaction among aligned large lamellar structures. M32/32 showed higher turbidity at 30 and $20^{\circ} \mathrm{C}$ in comparison to mixture solutions with other mixing ratios at same temperature because the mixed micelles in M32/32 contained larger fraction of lamellar structure. At 10 and $5^{\circ} \mathrm{C}, \mathrm{M} 32 / 32$ showed a similar tendency of increases in turbidity and viscosity with M36/28 due to the expansion and interaction in the lamellar structures in the solutions. It should be noted that the turbidity and viscosity for all of LPC/PC mixture solutions with different mixing ratios of LPC and PC changed reversibly against the change of temperature (within experimental range), reflecting the reversible structural changes of micelles with temperature dependence.

\section{Viscosity of aqueous wormlike micellar solutions}

The shear rate dependence of viscosity for mixture solutions with different mixing ratios of LPC and PC were measured at $20^{\circ} \mathrm{C}$ and plotted in Fig. 2. The viscosity $(\eta)$ of PC solution decreased with increasing shear rate $(\dot{\gamma})$ indicating the existence of lamellar structures in the solution, on the other hand, the viscosity of LPC solution was very low indicating the geometric preference of forming spherical micelle [16]. The viscosities of LPC/PC mixture solutions increased significantly suggesting the change of spherical micelles and lamellar structures to flexible elongated

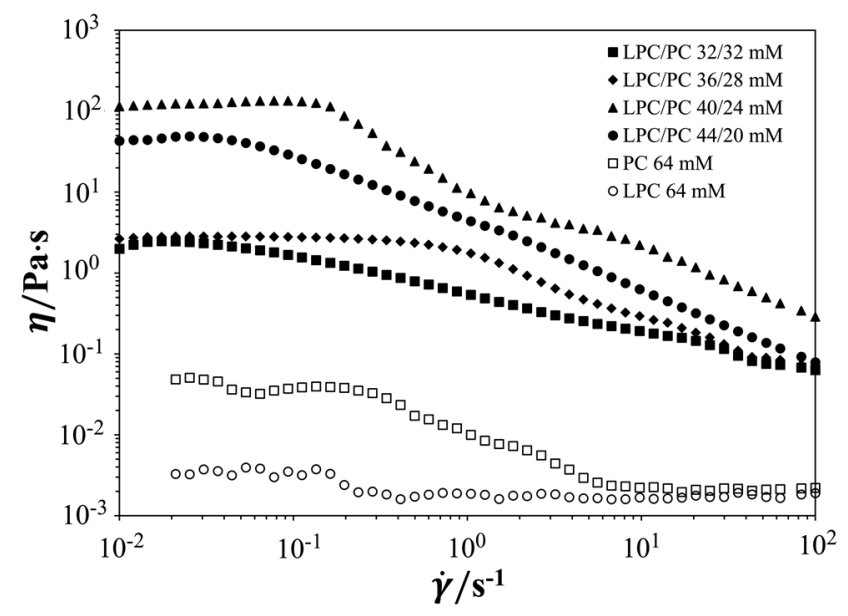

Fig. 2 A plot of viscosity $(\eta)$ of $64 \mathrm{mM} \mathrm{LPC/PC} \mathrm{mixture} \mathrm{solutions}$ with different mixing ratios $(32 / 32,36 / 28,40 / 24$ and 44/20) against shear rate $(\dot{\gamma})$ at $20^{\circ} \mathrm{C}$. 
micelles. These long and flexible wormlike micelles entangle with each other, and the gel network can be formed. The viscosities of M40/24 and M44/20 kept relatively high values and decreased under high shear rate showing shear thinning behavior. This behavior is frequently observed in the solution containing wormlike micelles where the wormlike micelles are thought to be aligned under high shear rate to decrease the entanglement [16-22]. The viscosities of M36/28 and M32/32 were lower than M40/24 and M44/20, and less enhanced shear thinning behaviors, suggesting that M36/28 and M32/32 contained a fraction of relatively short wormlike micelles, lamellar structures and spherical micelles as mixed micellar system.

The zero-shear viscosities $\left(\eta_{0}\right)$, for the LPC/PC mixture solutions were obtained by extrapolating the viscosity to the shear rate approaching to zero in Fig. 2, and plotted against the molar concentration of LPC $\left(C_{L P C}\right)$ in Fig. 3. $\eta_{0}$ for the LPC/PC mixture solutions were significantly higher than those for pure LPC and PC solutions at the same total concentration of $64 \mathrm{mM}$. $\eta_{0}$ was slightly increased with increasing $C_{L P C}$ from 32 to $36 \mathrm{mM}$ and a large increase with increasing $C_{L P C}$ from 36 to $40 \mathrm{mM}$ indicating the enhancement of the interaction and entanglement among micelles by forming the entangled wormlike micellar network [23-25]. Further increase of $C_{L P C}$ from 40 to $44 \mathrm{mM}, \eta_{0}$ decreased due to the disentanglement of wormlike micelles, and shortening or branching micelles [2, 26], then the spherical micelles of the LPC exceeded from the optimum mixing ratio for the formation of wormlike structure can be formed to coexist as the mixed micelles.

The change of viscosity in LPC/PC mixture solutions with different LPC/PC mixing ratios can be explained in terms of the critical packing parameter ( $c p p$ ). The cpp value of LPC is about $1 / 3$ which forms spherical micelles and shows low viscosity solution while PC has two alkyl chains with $c p p$ value $\sim 1$, and forms bilayers [1]. For a transition in the geometry to wormlike micelle, it requires the suitable

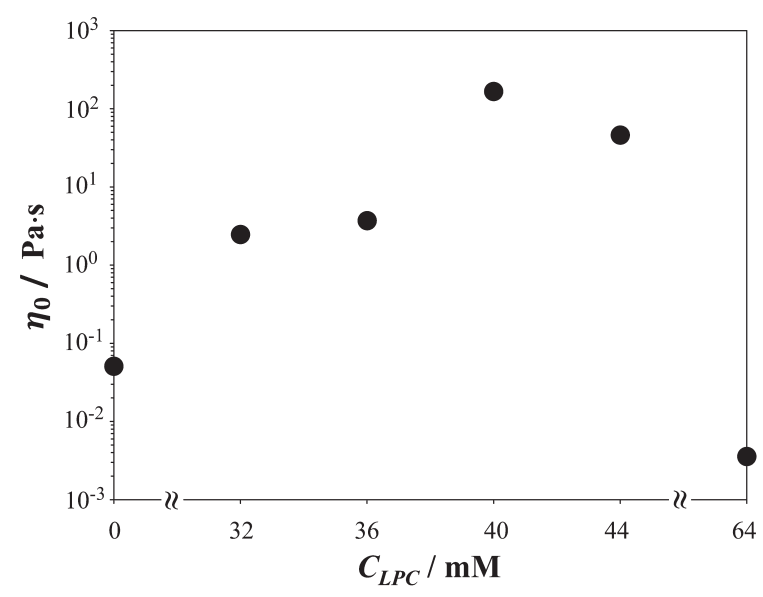

Fig. 3 Zero-shear viscosity $\left(\eta_{0}\right)$, of $64 \mathrm{mM} \mathrm{LPC/PC} \mathrm{mixture} \mathrm{solutions}$ with different mixing ratios $(32 / 32,36 / 28,40 / 24$ and 44/20) as a function of LPC molar concentrations at $20^{\circ} \mathrm{C}$. mixing ratio of different cpp molecules for average cpp of wormlike micelle which are varied from $1 / 3$ to $1 / 2$ [13]. Therefore, the suitable mixing ratio of LPC and PC is required for the formation of wormlike micelles.

\section{The viscoelasticity of wormlike micellar solutions}

For better understanding of viscoelastic behavior in the solutions which wormlike micelles formed by comicellization of the mixture of LPC and PC, oscillation frequency sweep measurements were carried out. Storage modulus $\left(G^{\prime}\right)$ and loss modulus $\left(G^{\prime \prime}\right)$ of different mixing ratios of LPC and PC mixture solutions were plotted against angular frequency $(\omega)$ at different temperatures in Fig. 4. M44/20 and M40/24, which were observed as transparent gel-like fluids at higher temperature and became slimy gels at low temperature in Fig. 1, showed liquid-like behavior $\left(G^{\prime \prime}>G^{\prime}\right)$ at a region of low $\omega$. With increasing $\omega, G^{\prime}$ increased and exceeded $G^{\prime \prime}$ at the crossover frequency $\left(\omega_{c}\right)$ and reached the plateau $\left(G_{0}\right)$ while $G^{\prime \prime}$ decreased after the crossover and increased again after reaching minimum. Both of M44/20 and M40/24 showed viscoelastic behavior in all temperatures with slight differences corresponding to the hardness of each mixture solution reflecting to a change of micellar structure in temperature dependence. On the other hand, M36/28 and M32/32 were observed as liquid-like behavior $\left(G^{\prime \prime}>G^{\prime}\right)$ at 35 and $30^{\circ} \mathrm{C}$. With decreasing temperature, M36/28 reverted viscoelastic response which exhibited predominant in elasticity at high $\omega$ region, while $G^{\prime}$ was slightly larger than $G^{\prime \prime}$ and almost parallel was observed in M32/32 with decreasing of slope when temperature decreased.

The mixture solutions showed viscoelastic behavior which arise from the entanglement of wormlike micelles. In this case, the viscoelastic behavior is expected to be explained by the Maxwell model [27]. The solid and dashed line were drawn on the result for M40/24 at $35^{\circ} \mathrm{C}$ in Fig. 4, which showed the viscoelastic behavior and observed as gel-like fluid. From the fitting data based on the Maxwell model with a single relaxation time $\left(\tau_{R}\right)$, the data fitted well at low $\omega$ region and there was discrepancy at high $\omega$ region due to the existence of entanglements of shorter time scales.

The relaxation time $\left(\tau_{R}\right)$ of wormlike micelle is related to the length of wormlike micelle, while $G_{0}$ is related to the density of the entanglement in the wormlike micellar network. The viscoelasticity behaviors based on the Maxwell model seen in M44/20 and M40/24 were not clearly observed for other mixture solutions at various temperatures. The crossing points of $G^{\prime}$ and $G^{\prime \prime}$ were observed for all mixture solutions, and the inverse of $\omega$ at the crossing point was considered to be related to the $\tau_{R}$. Regarding to the observation of viscosity in Fig. 1, we considered that the increase of $\omega$ at the crossing point with decreasing temperature for M44/20 came from the shortening of wormlike micelles. On the contrary, $\omega$ at the crossing point for M40/24 decreased with decreasing temperature from 35 to $20^{\circ} \mathrm{C}$ which was thought to be 


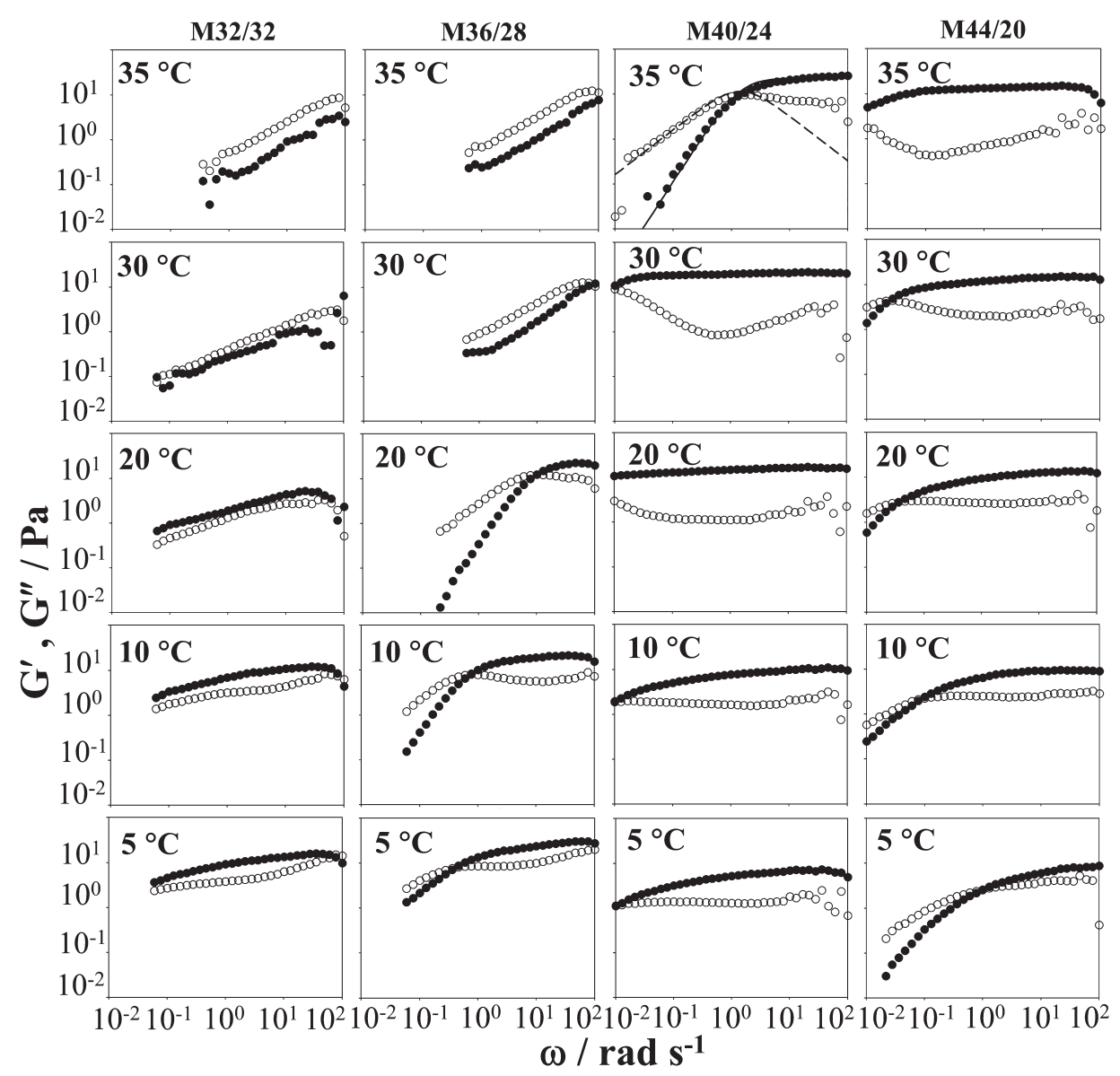

Fig. 4 The storage modulus ( $G^{\prime}$, filled symbols) loss modulus ( $G^{\prime \prime}$, opened symbols) as a function of angular frequency $(\omega)$ of $64 \mathrm{mM} \mathrm{LPC/PC} \mathrm{mixture}$ solutions with different mixing ratios (32/32, 36/28, 40/24 and 44/20) at different temperatures. Lines show the fitting data with a single Maxwell model.

corresponding to longer relaxation time of the entangled wormlike micelles, but $\omega$ at the crossing points increased with decreasing temperature to $5^{\circ} \mathrm{C}$, showing the loosening of the entanglement of wormlike micelles due to the shortening of wormlike micelles and/or co-existing of spherical micelles. For M36/28, $\omega$ at the crossing points decreased from 20 to $5^{\circ} \mathrm{C}$ indicating more structured of large lamellar structures with decreasing temperature. M32/32 was thought to be mixed micellar system which composed of different micellar structures. However, M32/32 was considered as the mixture of lamellar structures, short wormlike micelles and spherical micelles, indeed, there are large discrepancies from the Maxwell model indicating complicated relaxation mechanisms by lamellar structures and wormlike micelles as mixed micellar system.

The plateau modulus $\left(G_{0}\right)$ of M40/24 at 35 to $20^{\circ} \mathrm{C}$ were slightly larger than M44/20 indicating that the structure of gels formed by entangled wormlike micelles in M40/24 were the most stable which coinciding with the shear viscosity results in Fig. 2.

In order to clarify the existence of wormlike structures, we measured the dependence of $G_{0}$ on the total concentration of LPC and PC $\left(C_{\text {total }}\right)$ for the mixture solutions with the ratio of $\mathrm{LPC} / \mathrm{PC}=1.67$ as same with the ratio of $\mathrm{M} 40 / 24$

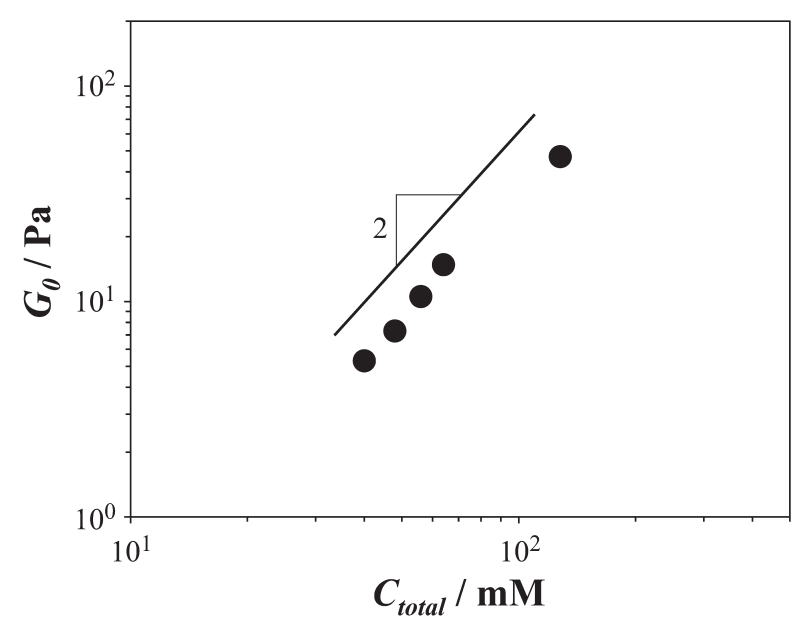

Fig. 5 Log-log plot of plateau modulus $\left(G_{0}\right)$ against $C_{\text {total }}$ for the mixture solutions with the ratio of LPC and PC of 1.67 at $20^{\circ} \mathrm{C}$.

which showed the typical viscoelastic behavior as the solution contained wormlike structures. The $C_{\text {total }}$ dependence of $G_{0}$ was indicated in a log-log plot in Fig. 5. Data points seem to increase linearly with a slope close to 2 in the plot. The viscoelasticity of solutions with wormlike micelles arise from the entanglement among wormlike micelles 
which shows the relationship of $G_{0} \sim C_{\text {total }}^{2}$ as observed in the entangled wormlike micelle of other systems [2,28]. The result in Fig. 5 followed this relationship suggesting the existence of long wormlike micelles in the mixture solution with the ratio of $\mathrm{LPC} / \mathrm{PC}=1.67$, which also supports the existence of wormlike micelle solutions with different ratios of LPC/PC though they might be mixtures of spherical, lamellar and short wormlike micelles.

\section{Conclusions}

In this work, we elucidated the formation of wormlike micelles in the mixture solutions with two different geometric amphiphiles of LPC and PC by heating. The mixture solutions with the ratio of $\mathrm{LPC} / \mathrm{PC}=1.67$ showed the highest viscosity and elasticity, suggesting the formation of long wormlike micelles attained by the average favorable cpp around 0.5 in the mixture. This consideration was supported by the dependence of $G_{0}$ on the total concentration of LPC and PC $\left(C_{\text {total }}\right) ; G_{0} \sim C_{\text {total }}$. The mixture solutions with the ratio lower than 1.67 (M32/32, M36/28) showed turbidity and the viscoelastic behavior which could be attributed to the interaction between lamellar phase of PC, spherical micelle of LPC and wormlike micelle of LPC/PC. On the other hand, the mixture solutions with the ratio higher than 1.67 (M44/20) was transparent and showed lower viscosity and elasticity than M40/24 because of shortening of wormlike micelles and/or co-existing of spherical micelles. It should be noted that M40/24 showed decreases of viscosity and the relaxation time in entanglement of wormlike micelles with decreasing temperature and this change was reversibly observed in the temperature range from 5 to $35^{\circ} \mathrm{C}$ indicating the change of stable $c p p$ in the wormlike structure.

Acknowledgements The authors are most grateful to thanks the Kewpie Corporation (Tokyo, Japan) for providing materials for this research, and the Kieikai Research Foundation for the financial support.

\section{References}

1. Israelachvili JN. Intermolecular and surface forces: 3 rd ed. Massachusetts: Academic Press; 2011.

2. Cates ME, Candau SJ. Statics and dynamics of worm-like surfactant micelles. J Phys Condens Matter. 1990; 2: 6869-93.

3. Grillo I, Penfold J. Self-assembly of mixed anionic and nonionic surfactants in aqueous solution. Langmuir. 2011; 27: 7453-63.

4. Svenson S. Controlling surfactant self-assembly. Curr Opin Colloid Interface. 2004; 9: 201-12.

5. Pichot R, Watson RL, Norton IT. Phospholipids at the interface: Current trends and challenges. Int J Mol Sci. 2013; 14: 11767-94.

6. Segota S, Tezak D. Spontaneous formation of vesicles. Adv Colloid Interface Sci. 2006; 121: 51-75.

7. Kumar VV. Complementary molecular shapes and additivity of the packing parameter of lipids. Proc Natl Acad Sci USA. 1991; 88: 444-8.
8. Hammad MA, Müller BW. Increasing drug solubility by means of bile salt-phosphatidylcholine-based mixed micelles. Eur J Pharm Biopharm. 1998; 46: 361-7.

9. Rupp C, Steckel H, Müller BW. Mixed micelle formation with phosphatidylcholines: the influence of surfactants with different molecule structures. Int J Pharm. 2010; 387: 120-8.

10. Ollivon M, Lesieur S, Grabielle-Madelmont C, Paternostre M. Vesicle reconstitution from lipid-detergent mixed micelles. Biochim Biophys Acta. 2000; 1508: 34-50.

11. Vinson PK, Talmon Y, Walter A. Vesicle-micelle transition of phosphatidylcholine and octyl glucoside elucidated by cryotransmission electron microscopy. Biophys J. 1989; 56: 669-81.

12. Morris D AN, McNeil R, Castellino FJ, Thomas JK. Interaction of lysophosphatidylcholine with phosphatidylcholine bilayers. Biochim Biophys Acta. 1980; 599: 380-90.

13. Fafaungwithayakul N, Klinkesorn U, Brenner T, Vichakacharu N, Matsukawa S. Thermally induced gelation of mixed phosphatidylcholine aqueous solution containing wormlike micelle structure. Jpn J Food Eng. 2014; 15: 233-42.

14. Yang J. Viscoelastic wormlike micelles and their applications. Curr Opin Colloid Interface Sci. 2002; 7: 276-81.

15. Jeong B, Lee KM, Gutowska A, An YH. Thermogelling biodegradable copolymer aqueous solutions for injectable protein delivery and tissue engineering. Biomacromolecules. 2002; 3 : $865-8$.

16. Hoffmann H, Ulbricht W. Structure-performance relationships in surfactants. In: Esumi K, Ueno M, editors. Surfactant sci ser. New York: CRC Press; 1997. p. 285-323.

17. Lin Y, Qiao Y, Yan Y, Huang J. Thermo-responsive viscoelastic wormlike micelle to elastic hydrogel transition in dual-component systems. Soft Matter. 2009; 5: 3047-53.

18. Rehage H, Hoffmann H. Viscoelastic surfactant solutions: model systems for rheological research. Mol Phys. 1991; 74: 933-73.

19. Ezrahi S, Tuval E, Aserin A. Properties, main applications and perspectives of worm micelles. Adv Colloid Interface Sci. 2006; 128-130: 77-102.

20. Hoffmann H, Ebert G. Surfactants, micelles and fascinating phenomena. Angew Chem Int Ed Engl. 1988; 27: 902-12.

21. Hoffmann H. Structure and flow in surfactant solutions. In: Herbs CA, Prud'homme RK, editors. ACS Symposium series 578. American Chemical Society; 1994. p. 2-31.

22. Acharya DP, Kunieda H. Wormlike micelles in mixed surfactant solutions. Adv Colloid Interface Sci. 2006; 123-126: 401-13.

23. Acharya DP, Varade D, Aramaki K. Effect of temperature on the rheology of wormlike micelles in a mixed surfactant system. J Colloid Interface Sci. 2007; 315: 330-6.

24. Sharma SC, Rodríguez C, Shrestha LK, Aramaki K. Short haired wormlike micelles in mixed nonionic fluorocarbon surfactants. J Colloid Interface Sci. 2007; 314: 223-9.

25. Aramaki K, Hoshida S, Arima S. Formation of wormlike micelles with natural-sourced ingredients (sucrose fatty acid ester and fatty acid) and a viscosity-boosting effect induced by fatty acid soap. Colloids Surf A Physicochem Eng Asp. 2012; 396: 278-82.

26. May S, Bohbot Y, Ben-Shaul A. Molecular theory of bending elasticity and branching of cylindrical micelles. J Phys Chem B. 1997; 101: 8648-57.

27. Cates ME. Reptation of living polymers: dynamics of entangled polymers in the reversible chain-scission reactions. Macromolecules. 1987; 20: 2289-96.

28. Shikata T, Hirata H, Kotaka T. Micelle formation of detergent molecules in aqueous media: viscoelastic properties of aqueous cetyltrimethylammonium bromide solution. Langmuir. 1987; 3: 1081-6. 